\title{
EHMTI-0162. Cognitive behavioural treatment for the chronic post-traumatic headache patient: a randomised controlled trial
}

\author{
D Kjeldgaard Nielsen ${ }^{1 *}, \mathrm{H}$ Forchhammer ${ }^{2}$, TW Teasdale $^{3}, \mathrm{RH}$ Jensen $^{1}$ \\ From 4th European Headache and Migraine Trust International Congress: EHMTIC 2014 \\ Copenhagen, Denmark. 18-21 September 2014
}

\section{Background}

Chronic post-traumatic headache $(\mathrm{CPTH})$ after mild head injury can be difficult to manage. Research is scarce and successful interventions are lacking.

\section{Aim}

To conduct a randomized controlled trial (RCT) exploring whether a group-based Cognitive Behavioural Therapy (CBT) intervention would lead to a relative decrease in headache, pain perception and psychological symptoms and an increase in quality of life in the study group compared to a waiting-list control group.

\section{Methods}

Ninety patients with CPTH according to ICHD-2 criteria were enrolled from the Danish Headache Center. Patients were randomly assigned to either a waiting- list group or to a nine-week CBT group intervention. At baseline and after 26 weeks all patients completed the Rivermead Postconcussion Questionnaire, SF-36, SCL-90-R (psychological distress) and a headache diary.

\section{Results}

The CBT had no effect on headache and only a minor impact on the CPTH patients' quality of life, psychological distress, and the overall experience of symptoms. The waiting-list group had also unchanged headache but, opposed to the treatment group, a significant decrease in somatic and cognitive symptoms indicating a spontaneous remission over time.

\section{Conclusions}

Our primarily negative findings confirm that management of patients with $\mathrm{CPTH}$ still remains a considerable challenge. Psychological group therapy with CBT might be effective in an earlier stage of CPTH and in less severely affected patients. There is an urgent need for development of new treatment strategies and a need for randomized controlled studies to test the efficacy of psychological therapy for CPTH patients.

No conflict of interest.

\section{Authors' details}

'Department of Neurology, Danish Headache Center University of Copenhagen, Glostrup, Denmark. ²Department of Neurology, University of Copenhagen, Glostrup, Denmark. ${ }^{3}$ The Department of Psychology, Faculty of Social Sciences University of Copenhagen, Copenhagen, Denmark.

Published: 18 September 2014

\section{doi:10.1186/1129-2377-15-S1-C29}

Cite this article as: Nielsen et al:: EHMTI-0162. Cognitive behavioural treatment for the chronic post-traumatic headache patient: a randomised controlled trial. The Journal of Headache and Pain 2014 15(Suppl 1):C29. 